\title{
The Effect of Foodsafe Level 1 Training on Inspection Report Results
}

\author{
Benjamin Duchen ${ }^{1}$, Helen Heacock ${ }^{2}$ \\ 1. Lead Author, B.Sc., B. Tech Student, School of Health Sciences, British Columbia Institute of Technology, 3700 Willingdon Ave, Burnaby, BC V5G $3 \mathrm{H} 2$ \\ 2. Supervisor, School of Health Sciences, British Columbia Institute of Technology, 3700 Willingdon Ave, Burnaby, BC V5G 3H2
}

\begin{abstract}
Food handlers equipped with food safety knowledge prevent foodborne illnesses. This study examined the relationship between worker Foodsafe level 1 training and critical violations reported on inspection results of non-chain restaurants in the Burnaby Fraser Health region. A total of 25 food service establishments with no critical violations on their routine inspections and 25 that had at least one critical violation participated in the telephone survey. Using the Mann-Whitney U two tailed t-test, it was shown that food service establishments with no critical violations on inspections had no significantly $(p=0.72)$ different proportions of Foodsafe level 1 trained staff than those with at least one critical violations on inspections. This study suggested that having more food handlers with food safety training does not impact how well restaurants score on inspections.
\end{abstract}

Keywords: Foodsafe, inspection, critical, violations.

\section{Introduction}

A Foodborne illness is defined as a human illness caused by a chemical, physical, or microbiological hazard with evidence indicating that food is the common source of exposure to the contamination (BC Center for Disease Control, 2012). Foodborne illnesses are a major concern in the field of public health. It is estimated that they cause one in eight Canadians to get sick every year (Public Health Agency of Canada, 2014). The annual incidence rate in British Columbia for foodborne illnesses is $20,150 / 100,000$ population and it translates to a $\$ 100$ million cost to the province every year from productivity loss and medical fees (Thomas et al., 2006).

Restaurant-associated foodborne illnesses are highly preventable through educated restaurant workers diligently practicing proper food handling techniques. The British Columbia government passed law in the Food Premises Regulation under the Public Health Act requiring mandatory food safety training. Section 10 of the regulation requires the operator of every food service establishment to be certified in the Foodsafe program and at least one employee on each shift must be Foodsafe certified in the absence of the operator (BC Ministry of Health, 2013). The main purpose of the program is to educate food handlers on how to prevent food borne illnesses. The Foodsafe level 1 course is targeted for front line food service employees and covers topics that deal with the top ten most common improper food handling practices that cause foodborne illnesses in North America (Calgary Health Region, 2002).

Environmental health officers of health authorities play a key role in safeguarding the public from restaurantassociated foodborne illnesses by conducting routine inspections to correct unsafe food handling practices that leads to foodborne illnesses (Island Health, 2013). During these inspections, environmental health officers have the responsibility of thoroughly going through the food service establishment to spot violations that compromises food safety, which are recorded on inspection reports. Critical violations are defined to be directly linked to foodborne illness outbreaks (BC Ministry of Health, 2006). Non-critical violations are less serious in nature and are not directly linked to outbreaks.

\section{Null and Alternative Hypotheses:}

$\mathbf{H}_{0}$ : There is no difference between the mean Foodsafe level 1 trained workers proportions in Burnaby food service establishments with and without a reported critical violation during their previous routine inspections. 
$\mathbf{H}_{\mathbf{a}}$ : There is a difference between the mean Foodsafe level 1 trained workers proportions in Burnaby food service establishments with and without a reported critical violation during their previous routine inspections.

\section{Literature Review}

Previous research has been conducted to evaluate the effectiveness of food safety training. One study evaluated the Foodsafe program and examined whether or not Foodsafe certification improves behavior from having increased knowledge. The researchers administered surveys via telephone to gather data on the knowledge and self-reported hand washing practices of food handlers with and without Foodsafe level 1 training. Food handlers with training reported significantly better hand washing knowledge and practices. The results of this study can be extrapolated to the other food safety topics, such as the ones covered in the Foodsafe training program, suggesting that food safety improves with Foodsafe training of food handlers if the knowledge is successfully applied in the food establishment workplace (McIntyre et al., 2013).

Another study was carried out in the United States comparing the routine inspection reports of chain restaurants requiring mandatory food safety training programs with non-chain restaurants that lack mandatory training in Florida during a one year period. The researchers found statistically significant differences in the number of critical violations between chain restaurants and independent restaurants, with chain restaurants averaging fewer critical violations (Murphy et al., 2011). Based on the findings, one would expect to find statically significant differences in the number of critical violations that are recorded on the routine inspection reports between restaurants with low and high percentages of Foodsafe trained food handlers.

On the other hand, there was evidence that supported the ineffectiveness of food safety training because knowledge does not always lead to behavior change. A study observed the behavior changes associated with food safety training. In this research, pre-training employees and post-training employees were examined by observation and by questionnaire surveys. The training program covered the main topics of the Foodsafe level 1 training program and can be seen as an equivalent. The researchers concluded that employees with food safety training had statistically significantly better knowledge and safer behavior only in the area of hand washing and not in other food handling areas (Roberts et al., 2008). The results suggested that training alone might not be enough to change behavior that improves food safety, with the exception of hand washing.

In the United States, food service establishments have the option of having their kitchen managers go through an extensive food safety training certification program. The topics covered in this training program are identical to the ones covered in Foodsafe program, also with the intention of preventing foodborne illnesses.

One study showed that establishments with a certified kitchen manager have significantly lower counts of critical violations in the areas of food handling, equipment cleaning and hot holding compared to establishments without a certified kitchen manager. The counts of critical violations on cooling, cooking temperatures and reheating are the same for restaurants with or without a certified kitchen manager (Cates et al., 2009). Another study took the research one step forward and examined the relationship between having a certified kitchen manager and the chance of a foodborne illness outbreak occurring. The results were that the major difference between outbreak and nonoutbreak restaurants was the presence of the certified kitchen manager. $71 \%$ of the non-outbreak restaurants had a certified kitchen manager while $29 \%$ of these restaurants did not have a certified kitchen manager. $32 \%$ of outbreak restaurants had a certified kitchen manager while $68 \%$ of them did not. Additionally, foodborne illness outbreaks that are caused by infected workers were not significantly related to whether or not the food service establishment had a certified kitchen manager or not, suggesting that the managers' training about worker sick policy is insufficient. Based on these two studies, support can be given that having one trained staff on the shift, in this case the certified kitchen manager, has significant impact on the reduction of critical violations on inspection reports and outbreak prevention. The results suggest that if the certified kitchen managers are sufficiently trained, they will ensure proper food handling procedures are followed to prevent foodborne illnesses and to prevent getting critical violations on the inspection reports (Hedberg et al., 2006). 
In summary, these studies suggest no significant difference in food safety between restaurants having varying percentages of food safety trained employees. The presence of a certified kitchen manager is enough to change the behavior of all the other staff, pointing to an absence and presence relationship between having a food safety trained worker and getting maximum food safety level. Having a certified kitchen manager in the American studies can be compared to having the operator needing to be Foodsafe trained in the BC regulations. The logic is that there is someone present with authority that has sufficient food safety knowledge to keep the establishment operations safe, preventing possible foodborne illnesses. However, one needs to consider the situation where the operator is absent. If the only employee with the Foodsafe training, required by the regulation, is not in a position to give orders to the coworkers, then he/she cannot help correct improper food handling techniques that lead to critical violations. In this scenario, it is no longer an absence and presence relationship, but is instead a linear relationship of getting better food safety as more employee are Foodsafe trained.

Since food handlers are human, one cannot discount psychological factors in the proposed study. There is a theory in psychology called the diffusion of responsibility that might affect the results and is defined as a person is less likely to take action if others are present (Ciccarelli \& White, 2009). When integrated into the proposed study, the phenomenon could occur if an employee of a food establishment observes an improper food handling method being done but does not take corrective action because he/she knows that the other coworkers are Foodsafe trained and should be able to step in to correct the error. On the other hand, if the employee knows he/she is the only person that is Foodsafe trained on the shift, he/she is more likely to take action to fix the problem. If the diffusion of responsibility plays a big role in decision making of food handlers, the number of critical violations on routine inspection reports can become inversely related to the percentage of food trained employees when results of the current study is analyzed.

There was no existing study that is exactly like the proposed study so it is difficult to predict the results from the literature review. There was a myriad of factors that affected the results of previous studies, such as the effectiveness of the Foodsafe training on behavioral changes of food handlers, the ability of Foodsafe trained workers to change others' behavior and the psychological phenomenon known as diffusion of responsibility.

\section{Methods}

The research was separated into two parts: (1) data collection of the proportion of Foodsafe trained employees using the survey method and, (2) the number of critical violations on the inspection report using existing data.

The first part of data collection involved accessing the Fraser Health online inspection reports to retrieve a comprehensive list of all food service establishments in the selected geographical area. The inclusion criteria for this study were all non-chain food service establishments in the Burnaby geographical area under the jurisdiction of Fraser Health with their routine inspection reports available online. Chain food service establishments were defined as more than two geographically separated food premises that are either owned by the same cooperation or were part of a franchise business (Glanz et al., 2007). Those that fitted the inclusion criteria were put into an excel spreadsheet. These food service establishments were further separated into two categories: ones that received no critical violations on their previous routine inspection report, designated as the NOCRIT group, and ones that received at least one critical violation, designated as the CRIT group. Both lists were individually put into the random list generator to randomize the order in which the food service establishments were contacted for a survey.

The second part of data collection was a randomized telephone survey of supervisors or managers to ask about their employee's training status. Proportions of employees with FoodSafe training was calculated for each FSE. All numerical data were recorded on the excel spreadsheet to the nearest five percent, organized by the name of the food service establishment. A total of 25 interviews for each FSE were collected during the afternoon period of 4:00PM to 7:00PM on weekdays to ensure survey administration remained consistent. The computer programs NCSS and Microsoft Excel were used to perform statistical analysis. A two sided t-test was used to analyze the data. 
All Food Service Establishments (FSEs)

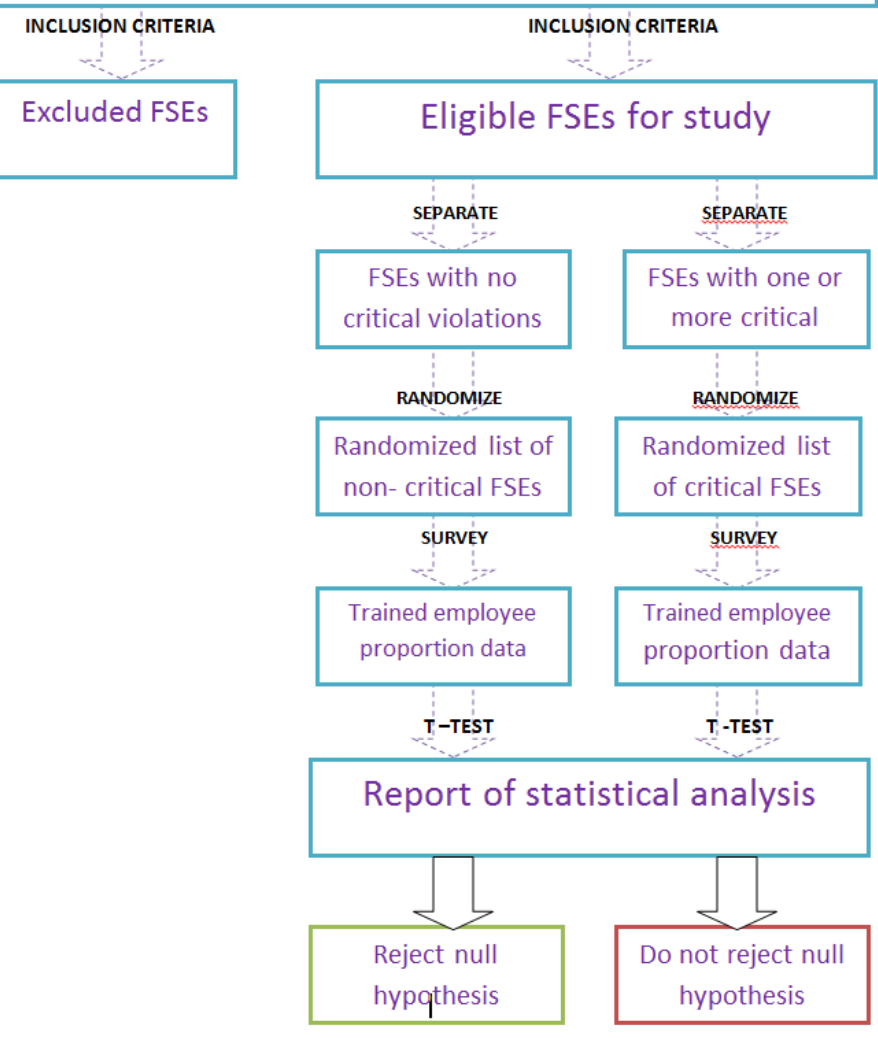

Fig.1. Visualization of the study methods.

\section{Results}

\section{Descriptive Statistics}

The data consisting of 25 data points in CRIT group and 25 data points in NOCRIT group was gathered were entered into the NCSS two-tailed $t$ test. The mean proportion of employees with FoodSafe certification in the CRIT group was 84.4 while the mean in the NOCRIT group was 85.6. The median of the CRIT group was 80.0 while the mean of the NOCRIT group was 90.0. It was found that that the range of the CRIT group was 50.0 and the range of the NOCRIT group was 40.0. The modes of both groups were 100.0. The standard deviation for the CRIT group was 14.6, and the standard deviation of the NOCRIT group was 13.3.

\begin{tabular}{|c|c|c|}
\hline & CRIT group & NOCRIT group \\
\hline Mean & 84.4 & 85.6 \\
\hline
\end{tabular}

\begin{tabular}{|c|c|c|}
\hline Median & 80.0 & 90.0 \\
\hline Range & 50.0 & 40.0 \\
\hline Mode & 100.0 & 100.0 \\
\hline Std Dev & 14.6 & 13.1 \\
\hline
\end{tabular}

Fig.2. Table containing descriptive statistics.

\section{Inferential Statistics}

From the NCSS program, it was determined that the data groups had equal variance but were not normally distributed. The correct t-test that was used to analyze this set of data was the non-parametric Mann-Whitney U or Wilcoxon Rank-sum t-test. According to this ttest, the null hypothesis cannot be rejected because the p-value was found to be 0.721 .

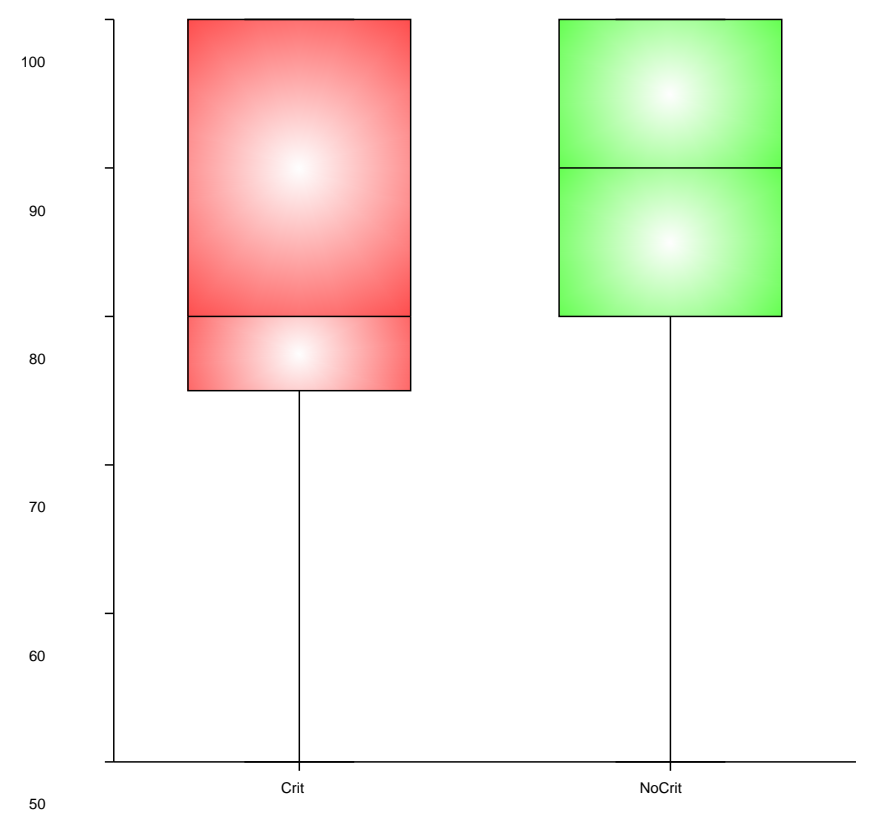

Fig.3. Box plot graph comparing data from the CRIT and NOCRIT data groups.

Since the p-value was greater than 0.05 , the null hypothesis cannot be rejected:

$\mathbf{H}_{0}$ : There is no difference between the mean Foodsafe level 1 trained workers proportions in Burnaby food service establishments with and without a reported 
critical violation during their previous routine inspections.

\section{Discussion}

The results were also not in agreement with some results found in studies in the literature review. McIntyre et al (2013), through a telephone survey, found out that Foodsafe level 1 trained workers reported significantly higher hand washing practices than those who were not trained. Logically, this would mean that food service establishments with more trained workers should have better inspection results on average because the environmental health inspectors would be likely to not observe improper and infrequent hand washing of those who are trained. The results did not support any evidence of this observation. Murphy et al. (2011) found that food service establishments that participated in mandatory food safety training programs, similar to Foodsafe, had significantly fewer critical violations on routine inspection results than those that did not participate. On the contrary, this study found no statically significant decrease in critical violations of restaurants with a higher Foodsafe trained employee to total employee ratio.

The results gathered from this study were also in agreement with some studies in the literature review. A questionnaire survey done by Roberts et al. (2008) examined the food safety knowledge and practices of pre-trained and post-trained food handlers. While it was found that post-trained food handlers had significantly better knowledge than pre-trained ones there was no difference in behavior that promotes good food safety. It was demonstrated that having good food safety knowledge, obtained from the food safety course similar to Foodsafe level 1, did not necessarily translate into better food handling practices. This result provided some insight into why this study showed that having more Foodsafe trained workers had no effect on inspection reports. Cates et al. (2009) and Hedberg et al. (2006) both conducted studies on certified kitchen managers, who are well trained in food safety knowledge. Cates et al. (2009) reported that restaurants with a certified kitchen manager had significantly lower counts of critical violations on routine inspection reports even in the presence of food handlers with no prior training. This result illuminates the possibility that having the one Foodsafe trained employee required by legislation is sufficient because that single employee is educating and instructing the other coworkers on how to handle food safely. Having more Foodsafe trained workers in the food service establishment would not be significantly different from having just the one trained worker. Hedberg et al. (2006) found that foodborne illness outbreaks were more likely to occur in restaurants without a certified kitchen manager. Again, the concept of having one Foodsafe trained worker produces maximum level of food safety, shown by the likelihood of foodborne illness outbreak resulting from the food service establishment. Investigation of these previous studies provided reasons for why study yielded results that did not have sufficient evidence to reject the null hypothesis.

There were also some observations noted in the data collection process that could have affected the results.

Firstly, the type of food service establishment could have played a factor in how infractions during inspections were interpreted. It seemed that lower risk food service establishments were graded more leniently than higher risk ones. Low risk establishments, such as bakeries and pizzerias, served food that had low inherent risk for foodborne illnesses and that had been cooked at a high temperature and for a long time to eliminate the same risk. High risk establishment, such as sushi restaurants, served food that had high inherent risk and that had no cooking step to kill potential pathogens. The same offence, such as general insufficient sanitation, noted in routine inspections was more likely to be regarded to be a critical violation in high risk establishments than low risk ones. Results were highly dependent on the food service establishment type in each of the establishments with no critical violations data group and those with critical violations data group.

Secondly, the voluntary nature of the study influenced the results. Establishments with low proportion of Foodsafe trained workers would be less inclined to participate and give data on their worker training status. The information is not volunteered because the food service establishments would want to protect their image. On the other hand, establishments with high proportions of Foodsafe trained workers would be more inclined to participate because they want to demonstrate their diligence of having foodsafe trained workers. The mode of both data groups were 100 and it showed evidence that the described phenomenon was 
occurring. Having less participation of establishments with low proportions of training and more participation of establishments with high proportions inflated the results and decreased the range of the data sets.

Thirdly, online inspection reports were not a perfect indication of food safety in the food service establishment. Inspections were all conducted by different inspectors so inspection reports cannot be compared to each other with exact precision. Some environmental health inspectors would focus on certain areas in the establishment such as the food preparation areas and could have missed critical violations found elsewhere. In addition, routine inspections were merely snapshots of food safety and were not related to the average levels. Inspectors could have dropped in a food service establishment when they were busy and noted several critical violations when it is normally very good with its food handling practices. These possibilities could have easily changed the data group of food service establishments that had no critical violations to the group with at least one critical violation.

Lastly, there was a minor discrepancy between the workers present at the time of routine inspections and the workers training status at the time of the survey done during this study. It could be the case that the workers were not Foodsafe trained at the time of the inspection and got trained before the telephone call was made to the food service establishment. This would increase the number of trained workers without changing the number of critical violations noted during a previous inspection. Also, the proportion between trained workers and total workers could have been inaccurately reflected during the routine inspection. For example, even if a food service establishment had 75\% Foodsafe trained food handlers employed, it could be the case that only one Foodsafe trained worker was present during the inspection. The rest of the workers present could be from the remaining $25 \%$ of the proportion and responsible for the critical violations recorded as a result of their improper food handling techniques.

\section{Conclusion}

The null hypothesis was accepted and alternate hypothesis was rejected because the p-value of the Mann-Whitney U t-test was 0.721 , which exceeds the threshold of 0.05 . This study suggests that the ratio of food handlers with Foodsafe level 1 training

\section{Limitations}

Design of the study could be drastically changed if the study could have spanned a longer time period. An alternative method of survey could have been utilized to increase participation rates because telephone survey participation rates in this study were low. The researcher could have been mistaken as a telemarketer. Having more time could also expand the study area and increase sample size, which increases the validity of the results.

\section{Future Research}

This study can be repeated with improvements to address the concerns of the discussed topics, such as having a larger sample size, categorizing different types of food service establishments into different data groups and using a different type of survey method and expanding the area of data collection. A larger sample of at least 100 data points for each group is required to produce meaningful results and an appropriate amount of time needs to be allocated for data collection. This change is to increase the power of the study and decrease the beta error if the null hypothesis is rejected again. Different types of food service establishment greatly influence the results so they should be categorized into groups depending on their risk levels: low, medium and high risk. A voluntary telephone survey was found to not be the optimal type of survey so an alternative one should be used for future repetitions. An online survey is recommended because it will increase participation rate due to the food service operators not feeling pressured or annoyed by telephone data collection. The area of the data sample can also be increased with the use of the online survey, resulting in better external validity of the study, allowing the results to be applied to a larger geographical area.

to total food handlers in a food service establishment does not affect its routine inspection results. Specifically, there is no evidence that Foodsafe training of workers would predict the absence or presence of critical violations noted on routine inspection. Based on the findings, section 10 of the Food Premises Regulation is sufficient when it 
requires only one employee to have Foodsafe training at any given time. More Foodsafe trained workers seemed to produce no additional beneficial effect. The resources of time and money spent to get additional workers trained would be wasted. The research question of "Is there a statically significant difference in the number of critical violations on routine inspection reports of food service establishments with varying percentages of Foodsafe level 1 trained employees” was answered with a no.

\section{Recommendation}

Even though this study does not give evidence that having more trained workers will improve food safety or inspection results, environmental health officers should still recommend Foodsafe level 1 training. The information provided in Foodsafe is crucial for preventing foodborne illness outbreaks. In addition, this training program covers many food handling requirements set in the Food Premises Regulation. One of the inspector's responsibilities during routine inspections is to educate food handlers on many of these requirements and how to achieve them. If the food handlers are Foodsafe certified, they can just be asked to review their course material and time can be allocated to address other problems.

A recommendation will be given to the Foodsafe organization to have an online information source to see which food handlers are Foodsafe certified. Certification date, certification level, name, and the place of employment are the pieces of information that will be posted on this database. This database would be helpful to the public when they decide where to dine. It could also be an incentive for food service establishments to ensure their workers are sufficiently trained.

\section{Acknowledgements}

This research paper is made possible through the guidance and teachings from the ENVH 3410 instructors, Helen Heacock and Bobby Sidhu.

\section{Competing Interest}

The authors declare that they have no competing interests.

\section{References}

BC Center for Disease Control. (2012). British

Columbia Foodborne Illness Outbreak Response

Protocol. Retrieved from

http://www.bccdc.ca/NR/rdonlyres/9891574D-

98DE-4538- 8B5A-

ADC2EBC2C88E/0/BCFIORP2009.pdf

BC Ministry of Health. (2013). Public Health Act: Food Premises Regulation. Retrieved from http://www.bclaws.ca/Recon/document/ID/freeside/ 11_210_99\#section10

BC Ministry of Health. (2006). Evidence Review:

Food Safety. Retrieved from

http://www.health.gov.bc.ca/public-

health/pdf/Food_Safety_Evidence_Review.pdf

Calgary Health Region. (2002). Top Improper Food

Handling Practices \& Common Job Hazards.

Retrieved from

http://www.calgaryhealthregion.ca/publichealth/env health/program_

areas/food_safety/documents/FSTop.pdf

Cates, S. C., Muth, M. K., Karns, S. A., Penne, M.

A., Stone, C. N., Harrison, J. E., \& Radke, V. J. (2009). Certified kitchen managers: do they improve restaurant inspection outcomes?. Journal of Food Protection, 72(2), 384-391.

Ciccarelli, S. K. \& White, J. N (2009). Psychology ( $2^{\text {nd }}$ ed.). Upper Saddle River, NJ: Pearson.

Foodsafe. (2009). Welcome. Retrieved from http://www.foodsafe.ca/

Fraser Health. (2014) Food Premises Facilities. Retrieved from http://www.healthspace.ca/fha/food

Glanz, K., Resnicow, K., Seymour, J., Hoy, K., Stewart, H., Lyons, M., \& Goldberg, J. (2007). How major restaurant chains plan their menus: the role of profit, demand, and health. American Journal of Preventive Medicine, 32(5), 383-388.

Hedberg, C. W., Smith, S. J., Kirkland, E., Radke, V., Jones, T. F., Selman, C. A., \& EHS-Net Working Group. (2006). Systematic environmental evaluations to identify food safety differences between outbreak and nonoutbreak 
restaurants. Journal of Food Protection, 69(11), 2697-2702.

Irwin, K., Ballard, J., Grendon, J., \& Kobayashi, J. (1989). Results of routine restaurant inspections can predict outbreaks of foodborne illness: the Seattle-King County experience. American Journal of Public Health, 79(5), 586-590.

Island Health. (2013). Inspections. Retrieved from http://www.viha.ca/mho/inspections/

McIntyre, L., Vallaster, L., Wilcott, L., Henderson, S. B., \& Kosatsky, T. (2013). Evaluation of food safety knowledge, attitudes and self-reported hand washing practices in FOODSAFE trained and untrained food handlers in British Columbia, Canada. Food Control, 30(1), 150-156.

Murphy, K. S., DiPietro, R. B., Kock, G., \& Lee, J. S. (2011). Does mandatory food safety training and certification for restaurant employees improve inspection outcomes? International Journal of Hospitality Management, 30(1), 150-156.
Public Health Agency of Canada. (2014). Estimates of Food-borne Illness in Canada. Retrieved from http://www.phac-aspc.gc.ca/efwd-emoha/efbiemoa-eng.php

Roberts, K. R., Barrett, B. B., Howells, A. D., Shanklin, C. W., Pilling, V. K., \& Brannon, L. A. (2008). Food safety training and foodservice employees' knowledge and behavior. Food Protection Trends, 28(4), 252-260.

Thomas, M. K., Majowicz, S., MacDougall, L., Sockett, P., Kovacs, S., Fyfe, M., Edge,V.L., Doré, K., Flint, J.A., Henson, S \& Jones, A. (2006). Population distribution and burden of acute gastrointestinal illness in British Columbia, Canada. BMC public health, 6(1), 307.

Vancouver Coastal Health. (2014). Food Establishment Listings by Facility Name. Retrieved from http://www.inspections.vcha.ca/Main

World Health Organization. (2014). Food Safety. Retrieved from http://www.who.int/topics /food_safety/en/ 